\title{
Algebraic treatment of the hypercoulomb problem
}

\author{
R. Bijker \\ Instituto de Ciencias Nucleares, \\ Universidad Nacional Autónoma de México, \\ Apartado Postal 70-543, 04510 México, D.F., México \\ F. Iachello \\ Center for Theoretical Physics, Sloane Laboratory, \\ Yale University, New Haven, CT 06520-8120, U.S.A. \\ E. Santopinto \\ Dipartimento di Fisica dell'Università di Genova, \\ Istituto Nazionale di Fisica Nucleare, Sezione di Genova, \\ via Dodecaneso 33, 16164 Genova, Italy
}

\begin{abstract}
A completely algebraic treatment of the six-dimensional hypercoulomb problem is discussed in terms of an oscillator realization of the dynamical algebra of $S O(7,2)$. Closed expressions are derived for the energy spectrum and form factors.
\end{abstract}




\section{Introduction}

Recently there has been renewed interest in the study of the three-body problem, mostly arising from constituent quark models of baryons in terms of three valence quarks. In these problems, the hypercentral approximation [1, which means using a hypercentral potential, provides often a good approximation to the actual situation 2, 3. 3. Among the hypercentral potentials, two play a special role: the six-dimensional harmonic oscillator and the six-dimensional coulomb potential, since both problems are exactly solvable. In this article, we present a completely algebraic solution of the hypercoulomb problem. The method is based on the use of $S O(7,2)$ as a dynamical group of the six-dimensional hypercoulomb potential [4. It is a generalization of $S O(4,2)$ dynamical group of the ordinary hydrogen atom [5].

In addition to obtaining a series of mathematical results associated with this rather complicated algebra, we briefly point out that the hypercoulomb potential can be used as a good approximation to actual physical situations. One occurs in atomic physics and it was mentioned long ago in [6]. The authors showed that the two-body coulomb potential in the hypercentral approximation, that is the hypercoulomb potential, provides a good description of the lower atomic states. Another application occurs in hadronic physics, in the constituent quark model of baryons. Due to the extraordinary difficulty to calculate physical quantities from QCD (in the nonperturbative regime) one has to rely on models, like constituent quark models. The short range behaviour is dominated by coulomb potentials as seen from the nonrelativistic reduction of the one-gluon exchange diagram [7] and the long range one by a linear potential as from results of lattice calculations. So one considers the potential dominated by a coulomb-like plus a linear confining term [2, 8, 9]. The coulomb like term provides a good approximation to the low-lying states and the effects of the linear confining terms plus other small contributions can be taken into account in perturbation theory as shown in [3]. This application is discussed in Section 8. Particular emphasis is paid to form factors which are the quantities of direct experimental interest, but which in general are very difficult to calculate.

\section{The hypercoulomb potential}

We consider the hypercoulomb potential in six dimensions

$$
H=\frac{p^{2}}{2 \mu}-\frac{\tau}{r}
$$

with $p^{2}=\sum_{j=1}^{6} p_{j}^{2}$ and $r^{2}=\sum_{j=1}^{6} r_{j}^{2}$. The energy eigenvalues and the degeneracy of the eigenstates can be obtained by studying the dynamical group $S O(7,2)$ 四. The generators of the Lie algebra of $S O(7,2)$ can be realized in coordinate space by

$$
\begin{aligned}
L_{j k} & =r_{j} p_{k}-r_{k} p_{j} \\
L_{j 7} & =\frac{1}{2} r_{j} p^{2}-p_{j} \sum_{k=1}^{6} r_{k} p_{k}+\frac{3}{2} i p_{j}-\frac{1}{2} r_{j}, \\
L_{j 8} & =\frac{1}{2} r_{j} p^{2}-p_{j} \sum_{k=1}^{6} r_{k} p_{k}+\frac{3}{2} i p_{j}+\frac{1}{2} r_{j}, \\
L_{j 9} & =r p_{j} \\
L_{78} & =\sum_{k=1}^{6} r_{k} p_{k}-\frac{5}{2} i \\
L_{79} & =\frac{1}{2}\left(r p^{2}-r\right), \\
L_{89} & =\frac{1}{2}\left(r p^{2}+r\right),
\end{aligned}
$$


with $j, k=1, \ldots, 6$. These generators are antisymmetric $L_{i j}=-L_{j i}$, and satisfy the commutation relations

$$
\left[L_{i j}, L_{k l}\right]=-i\left(g_{i k} L_{j l}+g_{j l} L_{i k}-g_{i l} L_{j k}-g_{j k} L_{i l}\right)
$$

with

$$
g_{i j}= \begin{cases}-\delta_{i j} & \text { for } j=1, \ldots, 7 \\ +\delta_{i j} & \text { for } j=8,9\end{cases}
$$

The $S O(7,2)$ algebra combines the $S O(2,1)$ spectrum generating algebra of the hypercoulomb problem and its $S O(7)$ degeneracy group into a single algebraic structure 狛. The corresponding subgroup chains are

$$
S O(7,2) \supset\left\{\begin{array}{ccc}
S O(6) & \otimes & S O(2,1) \\
\gamma & , & q \\
S O(7) & \otimes & S O(2) \\
\omega & , & q_{0}
\end{array}\right\} \supset S O(6) \otimes S O(2)
$$

The eigenstates of the hypercoulomb problem belong to a single infinite dimensional representation of $S O(7,2)$ 顿, which can be decomposed into the irreducible representations of its subgroups to provide a complete set of basis states. The generators and the quadratic Casimir operators of the groups appearing in the group lattice of Eq. (5) are given by

$$
\begin{array}{rlrl}
S O(7): & L_{j k}(j, k=1, \ldots, 7) & \Lambda_{7}^{2} & =\frac{1}{2} \sum_{j, k=1}^{7} L_{j k}^{2}, \\
S O(6): & L_{j k}(j, k=1, \ldots, 6) & \Lambda_{6}^{2}=\frac{1}{2} \sum_{j, k=1}^{6} L_{j k}^{2}, \\
S O(2,1): & T_{1}=L_{79}, T_{2}=L_{78}, T_{3}=L_{89} & T^{2}=T_{3}^{2}-T_{1}^{2}-T_{2}^{2}, \\
S O(2): & T_{3}=L_{89} & T_{3}^{2} .
\end{array}
$$

The Casimir operators satisfy the relations 4]

$$
\begin{aligned}
& T^{2}=\Lambda_{6}^{2}+\frac{15}{4}, \\
& T_{3}^{2}=\Lambda_{7}^{2}+\frac{25}{4} .
\end{aligned}
$$

This indicates that there exists a complementarity relationship 10 between the groups $S O(2,1)$ and $S O(6)$ within the single irreducible representation of $S O(7,2)$. As a consequence, the irreducible representations of $S O(6)$ are determined by those of $S O(2,1)$. The same holds for $S O(7)$ and $S O(2)$. The basis states $|\tilde{\psi}\rangle$ can be classified either by the $S O(2,1) \supset S O(2)$ labels $q, q_{0}$ which span the discrete representation $D^{+}$of $S O(2,1)$, or by the $S O(7) \supset S O(6)$ labels $\omega, \gamma$ which span the symmetric irreducible representation of $S O(7)$. They are simultaneous eigenfunctions of the Casimir operators of the subgroups in Eq. (5) and satisfy the eigenvalue equations

$$
\begin{aligned}
T^{2}|\tilde{\psi}\rangle & =q(q+1)|\tilde{\psi}\rangle, & & (q \text { real and }<0), \\
T_{3}|\tilde{\psi}\rangle & =q_{0}|\tilde{\psi}\rangle, & & \left(q_{0}=-q+s,\right. \text { with } \\
\Lambda_{7}^{2}|\tilde{\psi}\rangle & =\omega(\omega+5)|\tilde{\psi}\rangle, & & (\omega=0,1, \ldots), \\
\Lambda_{6}^{2}|\tilde{\psi}\rangle & =\gamma(\gamma+4)|\tilde{\psi}\rangle, & & (\gamma=0,1, \ldots, \omega) .
\end{aligned}
$$


Combining Eqs. (7) and (8), we find

$$
\begin{aligned}
q(q+1) & =\gamma(\gamma+4)+\frac{15}{4}, \\
q_{0}^{2} & =\omega(\omega+5)+\frac{25}{4},
\end{aligned}
$$

which, solving for $q<0$ and $q_{0} \geq-q$, gives

$$
q=-\gamma-\frac{5}{2}, \quad q_{0}=\omega+\frac{5}{2} .
$$

\section{Basis states}

For three identical particles, the hypercoulomb Hamiltonian of Eq. (11) is invariant under the permutation group $S_{3}$, and hence its eigenstates also carry good permutation symmetry $t=S, M$ or $A$, corresponding to the symmetric, mixed symmetric, and antisymmetric irreducible representation of $S_{3}$, respectively. In order to incorporate the permutation symmetry we associate the coordinates $r_{j}$ with the Jacobi vectors for the three-body problem [11]

$$
\begin{aligned}
& \vec{\rho}=\frac{1}{\sqrt{2}}\left(\vec{x}_{1}-\vec{x}_{2}\right) \equiv\left(r_{1}, r_{2}, r_{3}\right), \\
& \vec{\lambda}=\frac{1}{\sqrt{6}}\left(\vec{x}_{1}+\vec{x}_{2}-2 \vec{x}_{3}\right) \equiv\left(r_{4}, r_{5}, r_{6}\right) .
\end{aligned}
$$

Here $\vec{x}_{1}, \vec{x}_{2}$ and $\vec{x}_{3}$ denote the coordinates of the three particles. A convenient set of basis states is provided by the irreducible representations of the group chain

$$
\left|\begin{array}{ccccccccccc}
S O(7) & \supset & S O(6) & \supset & {[S U(3)} & \supset & S O(3) & \supset & S O(2)] & \otimes & S O(2) \\
\omega & , & \gamma & , & \left(n_{1}, n_{2}\right) & , & L & , & M_{L} & , & \nu
\end{array}\right\rangle .
$$

The reduction of $S O(6)$ can be obtained by using the complementarity relationship between the groups $S U(3)$ and $S O(2)$ within the symmetric irreducible representation $S O(6)$ [12]. As a consequence, the labels of $S U(3)$ are determined by that of $S O(2)$. The branching rules are given by

$$
\begin{aligned}
\gamma & =0,1, \ldots, \omega \\
\nu & =-\gamma,-\gamma+2, \ldots, \gamma, \\
\left(n_{1}, n_{2}\right) & =\left(\gamma, \frac{\gamma-\nu}{2}\right) .
\end{aligned}
$$

The reduction from $S U(3)$ to the rotation group $S O(3)$ is not fully reducible. In order to label the states uniquely an extra label is needed. We follow the procedure of [13] and introduce instead of $\left(n_{1}, n_{2}\right)$ the labels $(\lambda, \mu)=\left(n_{1}-n_{2}, n_{2}\right)=((\gamma+\nu) / 2,(\gamma-\nu) / 2)$. The values of $L$ contained in $(\lambda, \mu)$ are given by 113

$$
\begin{array}{rlrl}
\kappa & =\min \{\lambda, \mu\}, \min \{\lambda, \mu\}-2, \ldots, 1 \text { or } 0, & \\
L & = \begin{cases}\max \{\lambda, \mu\}, \max \{\lambda, \mu\}-2, \ldots, 1 \text { or } 0, & \text { for } \kappa=0, \\
\kappa, \kappa+1, \ldots, \kappa+\max \{\lambda, \mu\}, & \text { for } \kappa>0,\end{cases} \\
M_{L} & =-L,-L+1, \ldots, L . &
\end{array}
$$

The $S_{3}$ invariant states are given by the linear combinations

$$
\begin{aligned}
& \frac{-i}{\sqrt{2\left(1+\delta_{\nu, 0}\right)}}(|\nu\rangle-|-\nu\rangle), \\
& \frac{(-1)^{\nu_{0}}}{\sqrt{2\left(1+\delta_{\nu, 0}\right)}}(|\nu\rangle+|-\nu\rangle) .
\end{aligned}
$$


Here we have introduced the label $\nu_{0}$ by $\nu=\nu_{0}(\bmod 3)$. These wave functions transform for $\nu_{0}=0$ as $t=A, S$, and for $\nu_{0}=1,2$ as the two components of the mixed symmetric representation $t=M$. Summarizing, the basis states of the hypercoulomb problem can be characterized uniquely by

$$
|\tilde{\psi}\rangle=|\omega, \gamma,| \nu\left|, \kappa, L_{t}^{P}, M_{L}\right\rangle
$$

Here $P=(-)^{\gamma}$ denotes the parity. In Table 1 we present the classification scheme of the basis states for $\omega=4$.

\section{Energy spectrum}

The energy spectrum of the hypercoulomb problem in six dimensions can be obtained by using the properties of the $S O(2,1)$ spectrum generating algebra 4,14 . The Schrödinger equation can be expressed in terms of the generators of $S O(2,1)$ by introducing

$$
\mathcal{O}|\psi\rangle \equiv r(H-E)|\psi\rangle=0
$$

with

$$
\mathcal{O}=\left(\frac{1}{2 \mu}-E\right) T_{3}+\left(\frac{1}{2 \mu}+E\right) T_{1}-\tau .
$$

This equation can be simplified further by performing a rotation about a tilting angle $\theta$

$$
\tilde{\mathcal{O}}|\tilde{\psi}\rangle=0
$$

with

$$
\begin{aligned}
|\tilde{\psi}\rangle & =\mathcal{N} \mathrm{e}^{-i \theta T_{2}}|\psi\rangle \\
\tilde{\mathcal{O}} & =\mathrm{e}^{-i \theta T_{2}} \mathcal{O} \mathrm{e}^{i \theta T_{2}} \\
& =\left(\frac{1}{2 \mu}-E\right)\left(T_{3} \cosh \theta+T_{1} \sinh \theta\right)+\left(\frac{1}{2 \mu}+E\right)\left(T_{1} \cosh \theta+T_{3} \sinh \theta\right)-\tau
\end{aligned}
$$

The tilting angle may be chosen to diagonalize either the compact generator $T_{3}$ for the bound states, or the noncompact generator $T_{1}$ for the continuous states. The discrete spectrum is obtained by the choice

$$
\tanh \theta=\frac{E+\frac{1}{2 \mu}}{E-\frac{1}{2 \mu}}
$$

which reduces Eqs. (19) and (20) to an eigenvalue equation of $T_{3}$

$$
\left(\sqrt{-2 E / \mu} T_{3}-\tau\right)|\tilde{\psi}\rangle=0
$$

The energy eigenvalues of the hypercoulomb problem are obtained by solving Eq. 22

$$
E=-\frac{\mu \tau^{2}}{2 n^{2}}, \quad\left(n=q_{0}=\omega+\frac{5}{2}\right) .
$$

The corresponding tilting angle is given by

$$
\theta=-\ln (n / \mu \tau)
$$

The physical eigenstates, i.e. the solutions of the Schrödinger equation for the hypercoulomb problem, are related to the group states by a tilting rotation

$$
|\psi\rangle=\frac{1}{\mathcal{N}} \mathrm{e}^{-i \ln (n / \mu \tau) T_{2}}|\tilde{\psi}\rangle
$$


They satisfy the normalization condition (note that the group metric is $1 / r$ [15])

$$
\begin{aligned}
1=\langle\psi \mid \psi\rangle & =\frac{1}{\mathcal{N}^{2}}\left\langle\tilde{\psi}\left|\mathrm{e}^{i \ln (n / \mu \tau) T_{2}} r \mathrm{e}^{-i \ln (n / \mu \tau) T_{2}}\right| \tilde{\psi}\right\rangle \\
& =\frac{1}{\mathcal{N}^{2}}\left\langle\tilde{\psi}\left|\mathrm{e}^{i \ln (n / \mu \tau) T_{2}}\left(T_{3}-T_{1}\right) \mathrm{e}^{-i \ln (n / \mu \tau) T_{2}}\right| \tilde{\psi}\right\rangle \\
& =\frac{1}{\mathcal{N}^{2} \mu \tau}\left\langle\tilde{\psi}\left|n\left(T_{3}-T_{1}\right)\right| \tilde{\psi}\right\rangle \\
& =\frac{n^{2}}{\mathcal{N}^{2} \mu \tau} .
\end{aligned}
$$

Hence, the normalized physical eigenstates can be expressed in terms of the group states as

$$
|\psi\rangle=\frac{\sqrt{\mu \tau}}{n} \mathrm{e}^{-i \ln (n / \mu \tau) T_{2}}|\tilde{\psi}\rangle
$$

\section{$5 \quad$ Wave functions}

The wave function $|\tilde{\psi}\rangle$ satisfies the eigenvalue equations of Eq. (8), and hence is a $S O(2,1)$ eigenstate $|\tilde{\psi}\rangle=\left|q, q_{0}\right\rangle$ with

$$
\begin{aligned}
& \left|q, q_{0}\right\rangle=\sqrt{\frac{(-2 q-1) !}{\left(q_{0}+q\right) !\left(q_{0}-q-1\right) !}}\left(T_{+}\right)^{q_{0}+q}|q,-q\rangle, \\
& \left\langle q, q_{0}\right|=\sqrt{\frac{(-2 q-1) !}{\left(q_{0}+q\right) !\left(q_{0}-q-1\right) !}}\langle q,-q|\left(T_{-}\right)^{q_{0}+q},
\end{aligned}
$$

with $T_{ \pm}=T_{1} \pm i T_{2}$. The action of $T_{3}$ and the raising and lowering operators $T_{ \pm}$on the $S O(2,1)$ eigenstates $\left|q, q_{0}\right\rangle$ is given by 16

$$
\begin{aligned}
T_{3}\left|q, q_{0}\right\rangle & =q_{0}\left|q, q_{0}\right\rangle, \\
T_{ \pm}\left|q, q_{0}\right\rangle & =\sqrt{-\left(q \mp q_{0}\right)\left(q \pm q_{0}+1\right)}\left|q, q_{0} \pm 1\right\rangle .
\end{aligned}
$$

The ground state of the hypercoulomb potential has $\omega=0$, and hence the principal quantum number is $n=q_{0}=\omega+5 / 2=5 / 2$. Moreover, since the only allowed value of $\gamma$ is $\gamma=0$, we have $q=-\gamma-5 / 2=$ $-5 / 2$. Excited states with the same value of $q$, but with different values of $q_{0}$, can be created by applying the step operators $T_{ \pm}$according to Eq. (28). Since $T_{ \pm}$commute with the generators of $S O(6)$ (and its subgroups), these operators do not change the quantum numbers associated with the irreducible representations of $S O(6)$ and its subgroups $\gamma,|\nu|, \kappa, L_{t}^{P}$ and $M_{L}$. The operators $T_{ \pm}$are step operators in $q_{0}$, and hence change the value of the $S O(7)$ label $\omega$ by one unit. Excited states with the same value of $q_{0}$, but with different values of $q$, can be created by using the operators $L_{j 7}$ with $j=1, \ldots, 6$. Since $L_{j 7}$ is a generator of $S O(7)$, it does not change the value of $\omega$. However, under $S O(6)$ it transforms as a six-dimensional vector, and hence changes the value of $\gamma$ by one unit. The transformation properties of $T_{ \pm}$and $L_{j 7}$ under $S O(2,1)$ give rise to the selection rules

$$
\begin{aligned}
& T_{ \pm}: \quad \Delta q=\Delta \gamma=0, \quad \Delta q_{0}=\Delta \omega= \pm 1, \\
& L_{j 7}: \quad \Delta q=\Delta \gamma= \pm 1, \quad \Delta q_{0}=\Delta \omega=0 \text {. }
\end{aligned}
$$

Summarizing, the $S O(2,1)$ states $|\tilde{\psi}\rangle=\left|q, q_{0}\right\rangle$ can be created from the ground state by applying the operators $T_{+}$and $L_{j 7}$

$$
\begin{aligned}
& \left|-\frac{5}{2}, \frac{5}{2}\right\rangle \stackrel{T_{+}}{\longrightarrow}\left|-\frac{5}{2}, \frac{7}{2}\right\rangle \stackrel{T_{+}}{\longrightarrow}\left|-\frac{5}{2}, \frac{9}{2}\right\rangle \stackrel{T_{+}}{\longrightarrow} \ldots
\end{aligned}
$$

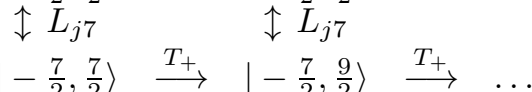



$$
\begin{aligned}
& \left|-\frac{9}{2}, \frac{9}{2}\right\rangle \stackrel{T_{+}}{\longrightarrow} \ldots
\end{aligned}
$$


Similarly, the ket states $\langle\tilde{\psi}|=\left\langle q, q_{0}\right|$ can be created by using the operators $T_{-}$and $L_{j 7}$. It is important to note, that the $S O(7)$ wave functions $|\tilde{\psi}\rangle=\left|q, q_{0}\right\rangle$ of Eq. (28) satisfy a different normalization condition, see Eq. (26), than the $S O(7)$ wave functions of [17, 18]. The hypercoulomb wave functions, i.e the eigenfunctions $|\psi\rangle$ of the original Hamiltonian of Eq. (1), correspond to the group states $|\tilde{\psi}\rangle$ tilted about $T_{2}$ by an angle $\theta=-\ln (n / \mu \tau)$, see Eq. (27). Since $T_{2}$ commutes with the generators of $S O(6)$ (and its subgroups), the hypercoulomb wave function $|\psi\rangle$ still has good $\gamma,|\nu|, \kappa, L_{t}^{P}$ and $M_{L}$, but the $S O(7)$ label $\omega$ no longer is a good quantum number.

\section{Oscillator realization}

The oscillator realization, that is frequently used for hydrogenlike problems characterized by the dynamical group $S O(4,2)$, is based on the isomorphism between $S O(4)$ and $S U(2) \otimes S U(2)$, and the Schwinger realization of $S U(2)$ [5]. However, this realization cannot be generalized to other dimensions, since it is based on a particular property of the three-dimensional case. In 19] an oscillator realization was proposed for the general $m$-dimensional case which is characterized by the dynamical group $S O(m+1,2)$. For six dimensions $(m=6)$ this gives a realization of the algebra of $S O(7,2)$ in terms of seven boson creation and annihilation operators

$$
\begin{aligned}
L_{j k} & =-i\left(a_{j}^{\dagger} a_{k}-a_{k}^{\dagger} a_{j}\right), \\
L_{j 8} & =\frac{1}{2} i\left(\sum_{k=1}^{7} a_{k}^{\dagger} a_{k}^{\dagger} a_{j}-2 \sum_{k=1}^{7} a_{j}^{\dagger} a_{k}^{\dagger} a_{k}-5 a_{j}^{\dagger}+a_{j}\right), \\
L_{j 9} & =-\frac{1}{2}\left(\sum_{k=1}^{7} a_{k}^{\dagger} a_{k}^{\dagger} a_{j}-2 \sum_{k=1}^{7} a_{j}^{\dagger} a_{k}^{\dagger} a_{k}-5 a_{j}^{\dagger}-a_{j}\right), \\
L_{89} & =\sum_{k=1}^{7} a_{k}^{\dagger} a_{k}+\frac{5}{2}, \quad(j, k=1, \ldots, 7) .
\end{aligned}
$$

The basis states of Eq. (16) correspond to linear combinations of the oscillator states

$$
\prod_{i=1}^{7} \frac{1}{\sqrt{n_{i} !}}\left(a_{i}^{\dagger}\right)^{n_{i}}|0\rangle,
$$

where the total number of oscillator quanta is $\sum_{i} n_{i}=\omega$. According to Eq. (11), we associate $a_{j}^{\dagger}$ with $j=1, \ldots, 6$ with the components of the two Jacobi vector boson operators and $a_{7}^{\dagger}$ with a scalar boson operator

$$
a_{k}^{\dagger} \equiv p_{\rho, k}^{\dagger}, \quad a_{k+3}^{\dagger} \equiv p_{\lambda, k}^{\dagger}, \quad a_{7}^{\dagger} \equiv s^{\dagger},
$$

with $k=1,2,3$. The oscillator realization of Eq. (32) is especially useful in deriving matrix elements.

\section{Form factors}

The six-dimensional hypercoulomb potential is an exactly solvable model, and hence provides a set of closed expressions for the spectrum and the form factors. The hypercoulomb ground state $\left|\psi_{0}\right\rangle=$ $\sqrt{\mu \tau} \mathrm{e}^{-i \ln \left(n_{0} / \mu \tau\right) T_{2}}\left|\tilde{\psi}_{0}\right\rangle / n_{0}$ is characterized by the principal quantum number $n_{0}=5 / 2$. According to the oscillator realization of Eq. (32), the ground state has zero oscillator quanta

$$
\left|\tilde{\psi}_{0}\right\rangle \equiv|0\rangle, \quad\left\langle\tilde{\psi}_{0}\right| \equiv\langle 0| .
$$

Excited states can be created by the procedure outlined in Section 5 in Eqs. (28) and (31). The oscillator realization of some $S O(2,1)$ eigenstates $\left|q, q_{0}\right\rangle$ and $\left\langle q, q_{0}\right|$ is given in Table 2 . 
The derivation of the elastic form factor requires the evaluation of the matrix element of $\exp \left(i k \lambda_{z}\right)=$ $\exp \left(i k r_{6}\right)$ in the ground state $\left|\psi_{0}\right\rangle$

$$
\begin{aligned}
F_{0}(k) & =\left\langle\psi_{0}\left|\mathrm{e}^{i k r_{6}}\right| \psi_{0}\right\rangle \\
& =\frac{\mu \tau}{n_{0}^{2}}\left\langle\tilde{\psi}_{0}\left|\mathrm{e}^{i \ln \left(n_{0} / \mu \tau\right) T_{2}} r \mathrm{e}^{i k r_{6}} \mathrm{e}^{-i \ln \left(n_{0} / \mu \tau\right) T_{2}}\right| \tilde{\psi}_{0}\right\rangle
\end{aligned}
$$

According to Eq.(2), both the coordinate $r_{j}$ and the radius $r$ can be expressed in terms of a linear combination of generators

$$
r_{j}=L_{j 8}-L_{j 7}, \quad r=L_{89}-L_{79} .
$$

This allows one to express $F_{0}(k)$ in terms of the generators of $S O(7,2)$

$$
\begin{aligned}
F_{0}(k) & =\frac{\mu \tau}{n_{0}^{2}}\left\langle\tilde{\psi}_{0}\left|\mathrm{e}^{i \ln \left(n_{0} / \mu \tau\right) L_{78}}\left(L_{89}-L_{79}\right) \mathrm{e}^{i k\left(L_{68}-L_{67}\right)} \mathrm{e}^{-i \ln \left(n_{0} / \mu \tau\right) L_{78}}\right| \tilde{\psi}_{0}\right\rangle \\
& =\frac{1}{n_{0}^{2}}\left\langle\tilde{\psi}_{0}\left|n_{0}\left(L_{89}-L_{79}\right) \mathrm{e}^{i k n_{0}\left(L_{68}-L_{67}\right) / \mu \tau}\right| \tilde{\psi}_{0}\right\rangle \\
& =\left\langle\tilde{\psi}_{0}\left|\mathrm{e}^{i k n_{0}\left(L_{68}-L_{67}\right) / \mu \tau}\right| \tilde{\psi}_{0}\right\rangle-\frac{1}{n_{0}}\left\langle\tilde{\psi}_{0}\left|L_{79} \mathrm{e}^{i k n_{0}\left(L_{68}-L_{67}\right) / \mu \tau}\right| \tilde{\psi}_{0}\right\rangle
\end{aligned}
$$

(i) The first term in Eq. (38) can be evaluated by rewriting

$$
\mathrm{e}^{i k n_{0}\left(L_{68}-L_{67}\right) / \mu \tau}=\mathrm{e}^{-i \alpha L_{67}} \mathrm{e}^{-i \beta L_{78}} \mathrm{e}^{-i \gamma L_{67}}
$$

with

$$
\begin{aligned}
\sinh (\beta / 2) & = \pm k n_{0} / 2 \mu \tau \\
\cosh (\beta / 2) & =\left(1+\left(k n_{0} / 2 \mu \tau\right)^{2}\right)^{1 / 2} \\
\sin \alpha & =-\sin \gamma= \pm \frac{1}{\cosh (\beta / 2)} \\
\cos \alpha & =-\cos \gamma=-\tanh (\beta / 2)
\end{aligned}
$$

Since the ground state has zero oscillator quanta, the matrix element of Eq. (39) reduces to

$$
\begin{aligned}
\left\langle\tilde{\psi}_{0}\left|\mathrm{e}^{i k n_{0}\left(L_{68}-L_{67}\right) / \mu \tau}\right| \tilde{\psi}_{0}\right\rangle & =\left\langle\tilde{\psi}_{0}\left|\mathrm{e}^{-i \alpha L_{67}} \mathrm{e}^{-i \beta L_{78}} \mathrm{e}^{-i \gamma L_{67}}\right| \tilde{\psi}_{0}\right\rangle \\
& =\left\langle\tilde{\psi}_{0}\left|\mathrm{e}^{-i \beta L_{78}}\right| \tilde{\psi}_{0}\right\rangle
\end{aligned}
$$

The ground state wave function satisfies

$$
\begin{aligned}
T_{3}\left|\tilde{\psi}_{0}\right\rangle & =n_{0}\left|\tilde{\psi}_{0}\right\rangle, \\
T_{-}\left|\tilde{\psi}_{0}\right\rangle & =a_{7}\left|\tilde{\psi}_{0}\right\rangle=0,
\end{aligned}
$$

and can be characterized by $\left|q=-n_{0}, q_{0}=n_{0}\right\rangle$. The ground state expectation value of $\exp \left(-i \beta L_{78}\right)$ in Eq. (41) is a representation matrix element for the discrete series $D^{+}$of $S O(2,1)$ [16]

$$
\left\langle\tilde{\psi}_{0}\left|\mathrm{e}^{-i \beta L_{78}}\right| \tilde{\psi}_{0}\right\rangle=\left\langle-n_{0}, n_{0}\left|\mathrm{e}^{-i \beta T_{2}}\right|-n_{0}, n_{0}\right\rangle=\left(\cosh \frac{\beta}{2}\right)^{-2 n_{0}}
$$

(ii) The second contribution to the form factor of Eq. (38) can be rewritten as

$$
\begin{aligned}
\left\langle\tilde{\psi}_{0}\left|L_{79} \mathrm{e}^{i k n_{0}\left(L_{68}-L_{67}\right) / \mu \tau}\right| \tilde{\psi}_{0}\right\rangle & =\left\langle\tilde{\psi}_{0}\left|L_{79} \mathrm{e}^{-i \alpha L_{67}} \mathrm{e}^{-i \beta L_{78}} \mathrm{e}^{-i \gamma L_{67}}\right| \tilde{\psi}_{0}\right\rangle \\
& =\left\langle\tilde{\psi}_{0}\left|\left(L_{79} \cos \alpha+L_{69} \sin \alpha\right) \mathrm{e}^{-i \beta L_{78}}\right| \tilde{\psi}_{0}\right\rangle
\end{aligned}
$$


The first term on the right hand side can be expressed in terms of the derivative of Eq. (43)

$$
\begin{aligned}
\left\langle\tilde{\psi}_{0}\left|L_{79} \mathrm{e}^{-i \beta L_{78}}\right| \tilde{\psi}_{0}\right\rangle & =-i\left\langle\tilde{\psi}_{0}\left|L_{78} \mathrm{e}^{-i \beta L_{78}}\right| \tilde{\psi}_{0}\right\rangle \\
& =\frac{d}{d \beta}\left\langle\tilde{\psi}_{0}\left|\mathrm{e}^{-i \beta L_{78}}\right| \tilde{\psi}_{0}\right\rangle \\
& =-n_{0}\left(\sinh \frac{\beta}{2}\right)\left(\cosh \frac{\beta}{2}\right)^{-2 n_{0}-1} .
\end{aligned}
$$

The second term in Eq. (44), proportional to $\sin \alpha$, vanishes identically. This can be seen by introducing the $S O(2,1) \otimes S O(2,1)$ algebra

$$
\begin{array}{lll}
{\left[G_{1}, G_{2}\right]=-i G_{3},} & {\left[G_{2}, G_{3}\right]=i G_{1},} & {\left[G_{3}, G_{1}\right]=i G_{2},} \\
{\left[H_{1}, H_{2}\right]=-i H_{3},} & {\left[H_{2}, H_{3}\right]=i H_{1},} & {\left[H_{3}, H_{1}\right]=i H_{2},} \\
{\left[G_{i}, H_{j}\right]=0,} &
\end{array}
$$

which is generated by the operators 19

$$
\begin{aligned}
G_{1} & =\frac{1}{2}\left(L_{68}+L_{79}\right), & H_{1} & =\frac{1}{2}\left(L_{68}-L_{79}\right), \\
G_{2} & =\frac{1}{2}\left(L_{78}-L_{69}\right), & H_{2} & =\frac{1}{2}\left(L_{78}+L_{69}\right), \\
G_{3} & =\frac{1}{2}\left(L_{67}+L_{89}\right), & H_{3} & =\frac{1}{2}\left(L_{67}-L_{89}\right) .
\end{aligned}
$$

We rewrite the second term in terms of the operators $G_{i}$ and $H_{j}$

$$
\begin{aligned}
\left\langle\tilde{\psi}_{0}\left|L_{69} \mathrm{e}^{-i \beta L_{78}}\right| \tilde{\psi}_{0}\right\rangle & =\left\langle\tilde{\psi}_{0}\left|\left(H_{2}-G_{2}\right) \mathrm{e}^{-i \beta\left(H_{2}+G_{2}\right)}\right| \tilde{\psi}_{0}\right\rangle \\
& =\left\langle\tilde{\psi}_{0}\left|H_{2} \mathrm{e}^{-i \beta H_{2}} \mathrm{e}^{-i \beta G_{2}}\right| \tilde{\psi}_{0}\right\rangle-\left\langle\tilde{\psi}_{0}\left|G_{2} \mathrm{e}^{-i \beta G_{2}} \mathrm{e}^{-i \beta H_{2}}\right| \tilde{\psi}_{0}\right\rangle .
\end{aligned}
$$

The action of $G_{3}$ and $H_{3}$ and the raising and lowering operators $G_{ \pm}=G_{1} \pm i G_{2}$ and $H_{ \pm}=H_{1} \pm i H_{2}$ on the ground state wave function can be evaluated by using the oscillator realization of Eq. (32) and Eq. (35)

$$
\begin{aligned}
G_{3}\left|\tilde{\psi}_{0}\right\rangle=-H_{3}\left|\tilde{\psi}_{0}\right\rangle & =\frac{n_{0}}{2}\left|\tilde{\psi}_{0}\right\rangle, \\
G_{-}\left|\tilde{\psi}_{0}\right\rangle=H_{+}\left|\tilde{\psi}_{0}\right\rangle & =0 .
\end{aligned}
$$

Thus, the ground state wave function is characterized by $g=-g_{0}=-n_{0} / 2$ and $h=h_{0}=-n_{0} / 2$. Eq. (49) shows that the matrix element of $\exp \left(-i \beta G_{2}\right)$ is a representation matrix element for the positive discrete series $D^{+}$, whereas that of $\exp \left(-i \beta H_{2}\right)$ corresponds to a representation matrix element for the negative discrete series $D^{-}$. The relevant matrix elements are given by [16]

$$
\left\langle-\frac{n_{0}}{2}, \frac{n_{0}}{2}\left|\mathrm{e}^{-i \beta G_{2}}\right|-\frac{n_{0}}{2}, \frac{n_{0}}{2}\right\rangle=\left\langle-\frac{n_{0}}{2},-\frac{n_{0}}{2}\left|\mathrm{e}^{-i \beta H_{2}}\right|-\frac{n_{0}}{2},-\frac{n_{0}}{2}\right\rangle=\left(\cosh \frac{\beta}{2}\right)^{-n_{0}} .
$$

Therefore, the two terms on the right hand side of Eq. (48) cancel, and the matrix element

$$
\left\langle\tilde{\psi}_{0}\left|L_{69} \mathrm{e}^{-i \beta L_{78}}\right| \tilde{\psi}_{0}\right\rangle=0 \text {, }
$$

vanishes identically.

Summarizing, the elastic form factor of Eq. (38) is obtained by combining the contributions from Eqs. (43), (45) and (51)

$$
F_{0}(k)=\left(\cosh \frac{\beta}{2}\right)^{-2 n_{0}-2}=\frac{1}{\left(1+\frac{25}{16 \mu^{2} \tau^{2}} k^{2}\right)^{7 / 2}},
$$


where we have used Eq. (40) and $n_{0}=5 / 2$ for the ground state wave function. This result is in agreement with [3, 20, in which the form factor was derived as an integral in bispherical coordinates. The derivation of transition form factors connecting the ground state to excited states proceeds in a similar way. The results are given in Table 3 .

The general result for the elastic form factor of the $m$-dimensional hypercoulomb potential can be obtained by using the properties of $S O(m+1,2)$ dynamical group. The derivation proceeds along the same lines as used in this section for $m=6$. The elastic form factor is given by

$$
F_{0}(k)=\frac{1}{\left(1+\frac{(m-1)^{2}}{16 \mu^{2} \tau^{2}} k^{2}\right)^{(m+1) / 2}},
$$

and shows a power-law dependence on $k$. For the six-dimensional case it reduces to Eq. (52).

\section{Nucleon form factors}

The hypercoulomb interaction whose properties have been described in the previous sections, can be used to analyze, at least approximately, nucleon phenomenology. In order to do so, one has first to assign the observed nucleon resonances to states of the hypercoulomb potential. This is done in Table 1 . The spatial wave function has to be combined with the spin-flavor and color parts, in such a way that the total wave function is antisymmetric (see e.g. [3, 21, 22). The nucleon itself is identified with the ground state $|\tilde{\psi}\rangle=\left|0,0,0_{S}^{+}\right\rangle$which has $n=5 / 2$, the Roper resonance is associated with $|\tilde{\psi}\rangle=\left|1,0,0_{S}^{+}\right\rangle$and $n=7 / 2$, and the negative parity resonances with $|\tilde{\psi}\rangle=\left|1,1,1_{M}^{-}\right\rangle$and $n=7 / 2$.

The formulas derived in the previous section provide a way to calculate form factors and helicity amplitudes between the ground state and the baryon resonances (see e.g. [2, 22, 231). As an example, we show in Figs. 1 and 2 the helicity amplitudes leading to the resonances $\mathrm{N}(1520) D_{13}$ and $\mathrm{N}(1535) S_{11}$. The hypercoulomb results are compared with experimental data and with complete calculations from [2] which take into account also the linear confining term. It is seen that the hypercoulomb results are comparable in quality with those of the second model [2]. Since the strength of the parameter of the linear confining term is not too strong, the hypercoulomb form factors provide a good lowest order approximation. We also comment on the fact that the hypercoulomb interaction produces form factors that decrease as the inverse of a power of the momentum transfer $k$, in contrast with the harmonic oscillator form factors (the other exactly solvable case) that drop too fast as a gaussian [23]. For example, the elastic form factor $G_{E}^{p}(k)$ behaves as

$$
G_{E}^{p}(k)= \begin{cases}1 /\left(1+k^{2} a^{2}\right)^{7 / 2} & \text { Hypercoulomb } \\ \exp \left(-k^{2} \beta^{2} / 6\right) & \text { Harmonic oscillator [23] }\end{cases}
$$

where $a^{2}=25 / 24 \mu^{2} \tau^{2}$ (see Table 3).

\section{Conclusions}

In this paper we have presented a completely algebraic treatment of the six-dimensional hypercoulomb problem in the context of the dynamical group $S O(7,2)$. We have developed a systematic way to construct the wave functions using step operators in the $S O(7) \supset S O(6)$ labels $\omega, \gamma$. This makes it possible to derive elastic form factors and transition matrix elements connecting the ground state to excited states in closed analytic form in an entirely algebraic way. In the derivation we have used an oscillator realization of the algebra of $S O(7,2)$. This procedure can readily be extended to the hypercoulomb problem in any number of dimensions, as it has been shown for the elastic form factor. We discussed briefly the application to baryon phenomenology, in which it was found that the form factors drop as powers of the momentum transfer, as it is observed experimentally. 
In conclusion, the present algebraic method provides an alternative to solving hypercoulomb problems with integro-differential methods.

\section{Acknowledgements}

This work is supported in part by DGAPA-UNAM under project IN101997 (R.B.), in part by DOE, Grant No. DE-FG02-91ER40608 (F.I.), and in part by INFN (E.S.).

\section{References}

[1] J. Ballot and M. Fabre de la Ripelle, Ann. of Phys. (N.Y.) 127, 62 (1980).

[2] M. Ferraris, M.M. Giannini, M. Pizzo, E. Santopinto and L. Tiator, Phys. Lett. B 364, 231 (1995); M. Aiello, M. Ferraris, M.M. Giannini, M. Pizzo and E. Santopinto, Phys. Lett. B 387, 215 (1996); M. Aiello, M.M. Giannini and E. Santopinto, J. Phys. G: Nucl. Part. Phys. 24, 753 (1998).

[3] E. Santopinto, F. Iachello and M.M. Giannini, Nucl. Phys. A 623, 101c (1997); E. Santopinto, F. Iachello and M.M. Giannini, Eur. Phys. J. A 1, 307 (1998).

[4] A.O. Barut and Y. Kitagawara, J. Phys. A: Math. Gen. 14, 2581 (1981).

[5] A.O. Barut and H. Kleinert, Phys. Rev. 156, 1541 (1967); ibid. 157, 1180 (1967); ibid. 160, 1149 (1967); Y. Nambu, Phys. Rev. 160, 1171 (1967).

[6] M. Fabre de la Ripelle and J. Navarro, Ann. Phys. (N.Y.) 123, 185 (1979).

[7] A. De Rújula, H. Georgi and S.L. Glashow, Phys. Rev. D 12, 147 (1975).

[8] E. Eichten, Phys. Rev. D 17, 3090 (1979); Phys. Rev. D 21, 203 (1980).

[9] J.-M. Richard and P. Taxil, Ann. Phys. (N.Y.) 150, 267 (1983).

[10] M. Moshinsky and C. Quesne, Phys. Lett. 29B, 482 (1969); J. Math. Phys. 11, 1631 (1970).

[11] P. Kramer and M. Moshinsky, Nucl. Phys. 82, 241 (1966).

[12] E. Chacón, O. Castaños and A. Frank, J. Math. Phys. 25, 1442 (1984).

[13] J.P. Elliott, Proc. Roy. Soc. A245, 128 (1958); Proc. Roy. Soc. A245, 562 (1958).

[14] B.G. Wybourne, 'Classical groups for physicists', Wiley, 1974.

[15] A.O. Barut and W. Rasmussen, J. Phys. B: Atom. Molec. Phys. 6, 1695 (1973).

[16] W.J. Holman and L.C. Biedenharn, Ann. Phys. 39, 1 (1966).

[17] R. Bijker and J.N. Ginocchio, Phys. Rev. C 45, 3030 (1992).

[18] E. Santopinto, R. Bijker and F. Iachello, J. Math. Phys. 37, 2674 (1996).

[19] E. Kyriakopoulos, Phys. Rev. 177, 2442 (1969).

[20] J. Leal Ferreira and P. Leal Ferreira, Lett. Nuovo Cimento 3, 43 (1970).

[21] N. Isgur and G. Karl, Phys. Rev. D 18, 4187 (1978); 192653 (1979); 201191 (1979).

[22] R. Bijker, F. Iachello and A. Leviatan, Ann. Phys. (N.Y.) 236, 69 (1994); Phys. Rev. C 54, 1935 (1996); Phys. Rev. D 55, 2862 (1997). 
[23] L.A. Copley, G. Karl and E. Obryk, Nucl. Phys. B13, 303 (1969).

[24] Particle Data Group, Phys. Rev. D54, 1 (1996).

[25] V. Burkert, Int. J. Mod. Phys. E1, 421 (1992) and private communication; G. Adams, N. Mukhopadhyay and P. Stoler, Excited Baryons 1988, proc. Topical Workshop, Troy, New York, 122 (1988). 
Table 1: Basis states of $S O(7)$ with $\omega=4$. The corresponding $S O(2,1)$ labels are $q_{0}=\omega+5 / 2$ and $q=-\gamma-5 / 2$.

\begin{tabular}{|c|c|c|c|c|}
\hline$\omega$ & $\gamma$ & $\nu$ & $\kappa$ & $L_{t}^{P}$ \\
\hline 4 & $\begin{array}{l}0 \\
1 \\
2\end{array}$ & $\begin{array}{r}0 \\
\pm 1 \\
\pm 2 \\
0 \\
\pm 3 \\
\pm 1 \\
\pm 4 \\
\pm 2 \\
0\end{array}$ & $\begin{array}{l}0 \\
0 \\
0 \\
1 \\
0 \\
1 \\
0 \\
1 \\
0 \\
2\end{array}$ & $\begin{array}{l}0_{S}^{+} \\
1_{M}^{-} \\
0_{M}^{+}, 2_{M}^{+} \\
1_{A}^{+}, 2_{S}^{+} \\
1_{S}^{-}, 1_{A}^{-}, 3_{S}^{-}, 3_{A}^{-} \\
1_{M}^{-}, 2_{M}^{-}, 3_{M}^{-} \\
0_{M}^{+}, 2_{M}^{+}, 4_{M}^{+} \\
1_{M}^{+}, 2_{M}^{+}, 3_{M}^{+}, 4_{M}^{+} \\
0_{S}^{+}, 2_{A}^{+} \\
2_{S}^{+}, 3_{A}^{+}, 4_{S}^{+}\end{array}$ \\
\hline
\end{tabular}


Table 2: Oscillator realization for the wave functions $|\tilde{\psi}\rangle=\left|q, q_{0}\right\rangle$ and $\langle\tilde{\psi}|=\left\langle q, q_{0}\right|$ with $\omega \leq 2$. For these states the labels $\nu$ and $\kappa$ are redundant.

\begin{tabular}{ccccccc}
\hline$q$ & $q_{0}$ & $\omega$ & $\gamma$ & $L_{t}^{P}$ & $M_{L}$ & oscillator \\
\hline$-\frac{5}{2}$ & $\frac{5}{2}$ & 0 & 0 & $0_{S}^{+}$ & 0 & $|0\rangle$ \\
$-\frac{5}{2}$ & $\frac{5}{2}$ & 0 & 0 & $0_{S}^{+}$ & 0 & $\langle 0|$ \\
$-\frac{5}{2}$ & $\frac{7}{2}$ & 1 & 0 & $0_{S}^{+}$ & 0 & $\frac{1}{\sqrt{5}}\langle 0| s$ \\
$-\frac{7}{2}$ & $\frac{7}{2}$ & 1 & 1 & $1_{M_{\rho}}^{-}$ & 1 & $\frac{i}{\sqrt{5}}\langle 0| p_{\rho, 1}$ \\
$-\frac{7}{2}$ & $\frac{7}{2}$ & 1 & 1 & $1_{M_{\lambda}}^{-}$ & 1 & $\frac{i}{\sqrt{5}}\langle 0| p_{\lambda, 1}$ \\
& & & & & & $\frac{1}{2 \sqrt{15}}\langle 0| s^{2}$ \\
$-\frac{5}{2}$ & $\frac{9}{2}$ & 2 & 0 & $0_{S}^{+}$ & 0 & $\frac{i}{\sqrt{35}}\langle 0| s p_{\rho, 1}$ \\
$-\frac{7}{2}$ & $\frac{9}{2}$ & 2 & 1 & $1_{M_{\rho}}^{-}$ & 1 & $\frac{i}{\sqrt{35}}\langle 0| s p_{\lambda, 1}$ \\
$-\frac{7}{2}$ & $\frac{9}{2}$ & 2 & 1 & $1_{M_{\lambda}}^{-}$ & 1 & $\frac{1}{2 \sqrt{35}}\langle 0|\left[p_{\rho, 1} p_{\rho, 1}+p_{\lambda, 1} p_{\lambda, 1}\right]$ \\
$-\frac{9}{2}$ & $\frac{9}{2}$ & 2 & 2 & $2_{S}^{+}$ & 2 & $\frac{1}{\sqrt{70}}\langle 0|\left[p_{\rho, 1} p_{\lambda, 0}-p_{\lambda, 1} p_{\rho, 0}\right]$ \\
$-\frac{9}{2}$ & $\frac{9}{2}$ & 2 & 2 & $1_{A}^{+}$ & 1 & $\frac{1}{\sqrt{35}}\langle 0| p_{\rho, 1} p_{\lambda, 1}$ \\
$-\frac{9}{2}$ & $\frac{9}{2}$ & 2 & 2 & $2_{M_{\rho}}^{+}$ & 2 & $\frac{1}{2 \sqrt{35}}\langle 0|\left[p_{\rho, 1} p_{\rho, 1}-p_{\lambda, 1} p_{\lambda, 1}\right]$ \\
$-\frac{9}{2}$ & $\frac{9}{2}$ & 2 & 2 & $2_{M_{\lambda}}^{+}$ & 2 & $\frac{1}{\sqrt{105}}\langle 0| p_{\rho} \cdot p_{\lambda}$ \\
$-\frac{9}{2}$ & $\frac{9}{2}$ & 2 & 2 & $0_{M_{\rho}}^{+}$ & 0 & $\frac{1}{2 \sqrt{105}}\langle 0|\left[p_{\rho} \cdot p_{\rho}-p_{\lambda} \cdot p_{\lambda}\right]$ \\
$-\frac{9}{2}$ & $\frac{9}{2}$ & 2 & 2 & $0_{M_{\lambda}}^{+}$ & 0 & \\
\hline & & & & & &
\end{tabular}


Table 3: Form factors for the hypercoulomb problem. The hypercoulomb wave functions are given by Eq. (27) $|\psi\rangle=\sqrt{\mu \tau} \mathrm{e}^{-i \ln (n / \mu \tau) T_{2}}|\tilde{\psi}\rangle / n$ with $|\tilde{\psi}\rangle=\left|\omega, \gamma, L_{t}^{P}\right\rangle$ and $n=\omega+5 / 2$. The initial state is $|\tilde{\psi}\rangle=\left|0,0,0_{S}^{+}\right\rangle$and $a=n_{0} / \sqrt{6} \mu \tau=5 / 2 \sqrt{6} \mu \tau$.

\begin{tabular}{c|c}
\hline$\left\langle\tilde{\psi}^{\prime}\right|=\left\langle\omega, \gamma, L_{t}^{P}\right|$ & $\left\langle\psi^{\prime}\left|\mathrm{e}^{i \sqrt{2 / 3} k r_{6}}\right| \psi\right\rangle$ \\
\hline$\left\langle 0,0,0_{S}^{+}\right|$ & $-i \sqrt{7}\left(\frac{5}{6}\right)^{4}\left(\frac{7}{6}\right)^{4} \frac{k a}{\left(1+\frac{49}{36} k^{2} a^{2}\right)^{9 / 2}}$ \\
$\left\langle 1,1,1_{M}^{-}\right|$ & $\sqrt{7}\left(\frac{5}{6}\right)^{4}\left(\frac{7}{6}\right)^{5} \frac{k^{2} a^{2}}{\left(1+\frac{49}{36} k^{2} a^{2}\right)^{9 / 2}}$ \\
$\left\langle 1,0,0_{S}^{+}\right|$ & $-\frac{\sqrt{21}}{\sqrt{2}}\left(\frac{5}{7}\right)^{5}\left(\frac{9}{7}\right)^{5} \frac{k^{2} a^{2}}{\left(1+\frac{81}{49} k^{2} a^{2}\right)^{11 / 2}}$ \\
$\left\langle 2,2,2_{S}^{+}\right|$ & $\frac{\sqrt{21}}{\sqrt{2}}\left(\frac{5}{7}\right)^{5}\left(\frac{9}{7}\right)^{5} \frac{k^{2} a^{2}}{\left(1+\frac{81}{49} k^{2} a^{2}\right)^{11 / 2}}$ \\
$\left\langle 2,2,2_{M}^{+}\right|$ & $-\frac{\sqrt{21}}{2}\left(\frac{5}{7}\right)^{5}\left(\frac{9}{7}\right)^{5} \frac{k^{2} a^{2}}{\left(1+\frac{81}{49} k^{2} a^{2}\right)^{11 / 2}}$ \\
$\left\langle 2,1,1_{M}^{-}\right|$ & $-i \frac{\sqrt{7}}{3}\left(\frac{5}{7}\right)^{4}\left(\frac{9}{7}\right)^{5} \frac{k a\left[1+\frac{486}{49} k^{2} a^{2}\right]}{\left(1+\frac{81}{49} k^{2} a^{2}\right)^{11 / 2}}$ \\
$\left\langle 2,0,0_{S}^{+}\right|$ & $\frac{\sqrt{3}}{2}\left(\frac{5}{7}\right)^{4}\left(\frac{9}{7}\right)^{5} \frac{k^{2} a^{2}\left[1+\frac{486}{49} k^{2} a^{2}\right]}{\left(1+\frac{81}{49} k^{2} a^{2}\right)^{11 / 2}}$ \\
\hline
\end{tabular}


Table 4: Identification of the states of Eq. 27) and Table 1 (the quantum numbers are $\omega, \gamma, L_{t}^{P}$ ) with the observed baryon resonances. The spin, $S$, and the total angular momentum and parity, $J^{P}$, is also indicated. The experimental masses are from 24].

\begin{tabular}{|c|c|c|c|c|c|}
\hline$\omega \gamma L^{P}$ & $S$ & $J^{P}$ & Baryon & Status & $M_{\text {exp }}(\mathrm{MeV})$ \\
\hline $000^{+}$ & \multirow{19}{*}{$\begin{array}{l}\frac{1}{2} \\
\frac{3}{2} \\
\frac{1}{2} \\
\frac{1}{2} \\
\frac{1}{2} \\
\frac{1}{2} \\
\mathbf{X} \\
\mathbf{X}\end{array}$} & \multirow{19}{*}{$\begin{array}{l}\frac{1}{2}^{+} \\
\frac{3}{2}^{+} \\
\frac{1}{2}^{+} \\
\frac{3}{2}^{+} \\
\frac{1}{2}^{-} \\
\frac{3}{2}^{-} \\
\frac{1}{2}^{-} \\
\frac{3}{2}^{-} \\
\frac{5}{2}^{-} \\
\frac{1}{2} \\
\frac{3}{2}^{-} \\
\frac{1}{2}^{+} \\
\frac{3}{2}^{+} \\
\frac{5}{2}^{+} \\
\frac{1}{2}^{+} \\
\frac{3}{2}^{+} \\
\frac{5}{2}^{+} \\
\frac{7}{2}^{+} \\
\frac{1}{2}^{-}\end{array}$} & $\mathrm{N}(938) P_{11}$ & $* * * *$ & 938 \\
\hline $000^{+}$ & & & $\Delta(1232) P_{33}$ & $* * * *$ & 1232 \\
\hline $100^{+}$ & & & $\mathrm{N}(1440) P_{11}$ & $* * * *$ & 1440 \\
\hline $100^{+}$ & & & $\Delta(1600) P_{33}$ & $* * *$ & 1600 \\
\hline $111^{-}$ & & & $\mathrm{N}(1535) S_{11}$ & $* * * *$ & 1535 \\
\hline $111^{-}$ & & & $\mathrm{N}(1520) D_{13}$ & $* * * *$ & 1520 \\
\hline $111^{-}$ & & & $\mathrm{N}(1650) S_{11}$ & $* * * *$ & 1650 \\
\hline $111^{-}$ & & & $\mathrm{N}(1700) D_{13}$ & $* * *$ & 1700 \\
\hline $111^{-}$ & & & $\mathrm{N}(1675) D_{15}$ & $* * * *$ & 1675 \\
\hline $111^{-}$ & & & $\Delta(1620) S_{31}$ & $* * * *$ & 1620 \\
\hline $111^{-}$ & & & $\Delta(1700) D_{33}$ & $* * * *$ & 1700 \\
\hline $200^{+}$ & & & $\mathrm{N}(1710) P_{11}$ & $* * *$ & 1710 \\
\hline $222^{+}$ & & & $\mathrm{N}(1720) P_{13}$ & $* * * *$ & 1720 \\
\hline $222^{+}$ & & & $\mathrm{N}(1680) F_{15}$ & $* * * *$ & 1680 \\
\hline $222^{+}$ & & & $\Delta(1910) P_{31}$ & $* * * *$ & 1910 \\
\hline $222^{+}$ & & & $\Delta(1920) P_{33}$ & $* * *$ & 1920 \\
\hline $222^{+}$ & & & $\Delta(1905) F_{35}$ & $* * * *$ & 1905 \\
\hline $222^{+}$ & & & $\Delta(1950) F_{37}$ & $* * * *$ & 1950 \\
\hline $211^{-}$ & & & $\Delta(1900) S_{31}$ & $* * *$ & 1900 \\
\hline
\end{tabular}




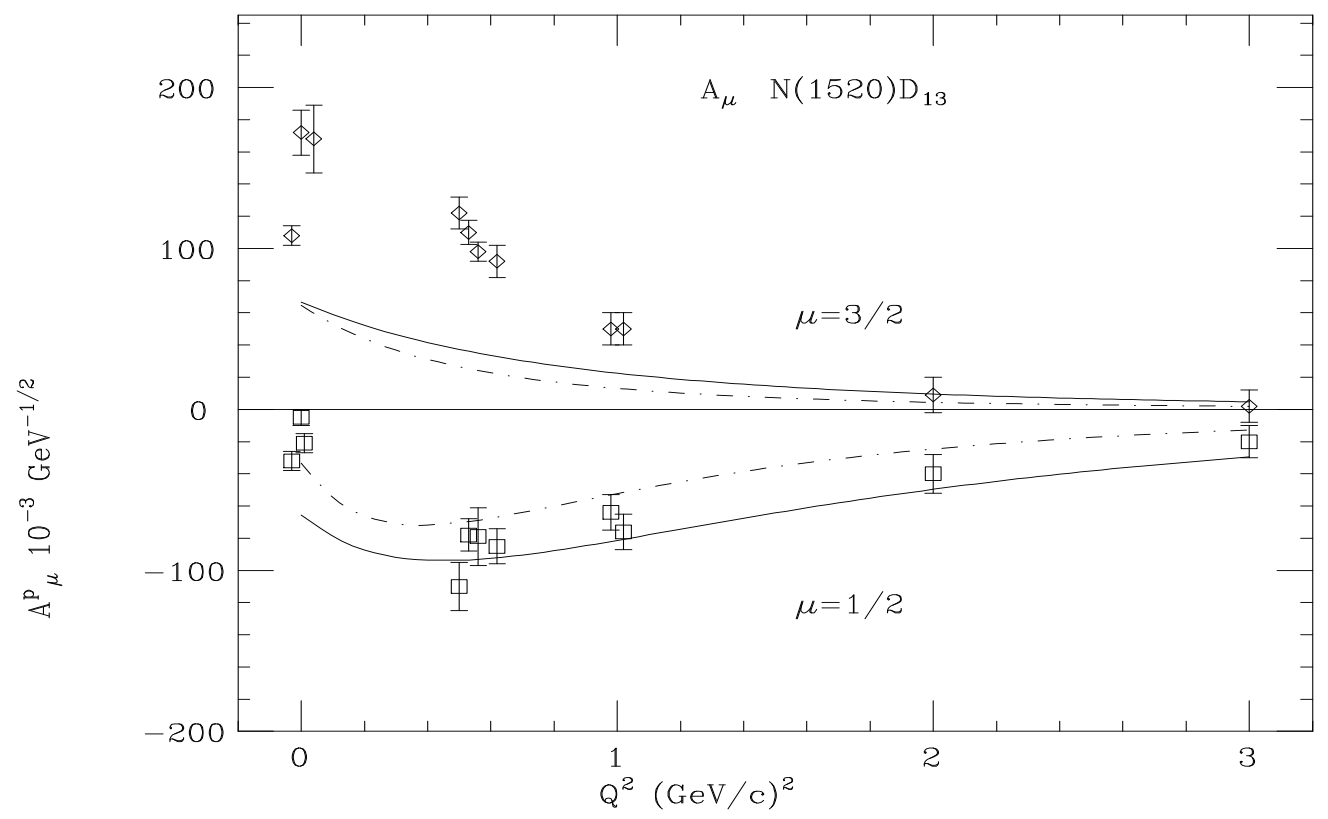

Figure 1: The helicity amplitudes $A_{1 / 2}^{p}$ and $A_{3 / 2}^{p}$ in the Breit frame for the excitation of the $\mathrm{N}(1520) D_{13}$ resonance. The full curve is the complete result of the model of [2], while the dashed curve is the result without the linear confining term. The experimental data are taken from 25]. 


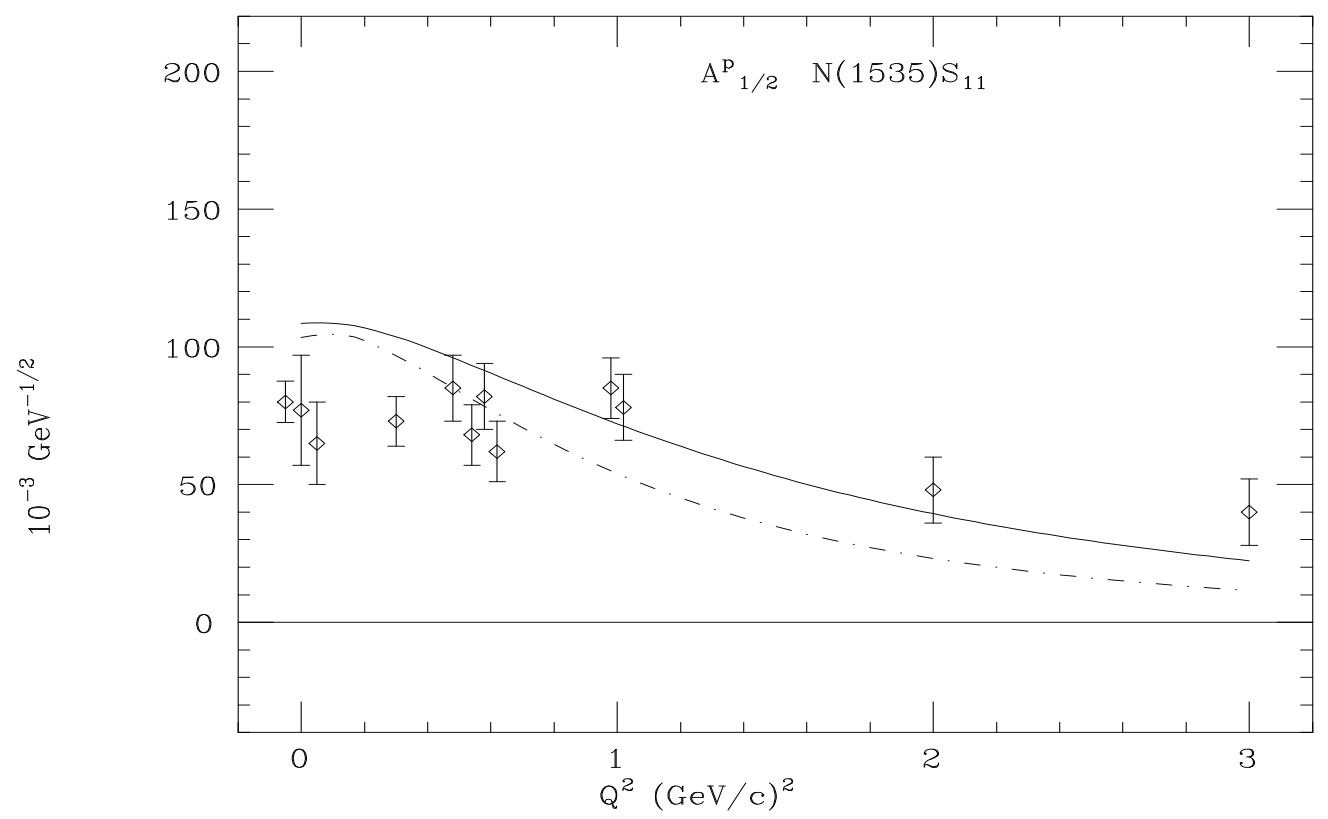

Figure 2: The same as in Fig. 1, but for the excitation of the N(1535) $S_{11}$ resonance. 